\title{
Exploring the Diabetic Gastroparesis Patient Experience: Patient Exit Interviews
}

\author{
Claire M. Ervin · David S. Reasner · Jennifer T. Hanlon · Sheri E. Fehnel
}

Received: July 14, 2017 / Published online: October 27, 2017

(C) The Author(s) 2017. This article is an open access publication

\begin{abstract}
Introduction: To improve understanding of the diabetic gastroparesis (DGP) patient experience and inform the patient-reported outcome measurement strategy for future trials in DGP, qualitative interviews were conducted with participants in a phase 2 clinical trial of a novel DGP treatment.

Methods: Trial participants were invited to participate in interviews at both the pretreatment visit (PTV) and the end-of-treatment visit (EOTV). The interviews were conducted by experienced qualitative researchers and followed a semistructured interview guide. The PTV interviews focused on patients' DGP symptoms and the impact of DGP on their lives, and the EOTV interviews focused on any symptom changes patients experienced during the trial.
\end{abstract}

Results: Of 90 enrolled trial participants, 78 $(86.7 \%)$ opted to participate in the interview

Enhanced Content To view enhanced content for this article go to http://www.medengine.com/Redeem/ 19CCF0602F009B7D.

C. M. Ervin $(\bowtie) \cdot$ S. E. Fehnel

RTI Health Solutions, Research Triangle Park,

NC, USA

e-mail: cervin@rti.org

D. S. Reasner · J. T. Hanlon

Ironwood Pharmaceuticals Inc, Cambridge, MA, USA study. Bloating, stomach fullness, upper abdominal pain, vomiting, constipation, and heartburn or reflux were each reported spontaneously by a majority of the 73 PTV interview participants with evaluable data. These patients commonly reported bloating $(n=20)$, upper abdominal pain $(n=12)$, and nausea $(n=11)$ as their most bothersome DGP symptom. Of 51 EOTV interview participants, 44 (86.3\%) reported improvement in at least one DGP symptom either spontaneously or when asked about specific symptoms reported during their PTV interview.

Conclusion: Bloating, abdominal pain, nausea, constipation, stomach fullness, vomiting, and heartburn were frequently reported by patients as the most bothersome and important-to-treat symptoms. These results support the assessment of these symptoms in future DGP clinical trials, whether for symptom improvement or worsening. Funding: Ironwood Pharmaceuticals.

Trial Registration: ClinicalTrials.gov identifier NCT02289846.

Keywords: Exit interviews; Gastroenterology; Gastroparesis; Patient interviews; Patient-centric; Patient-reported; Qualitative

\section{INTRODUCTION}

Gastroparesis is defined as delayed gastric emptying, in the absence of mechanical 
obstruction, that may cause nausea, vomiting, and other upper gastrointestinal (GI) tract symptoms $[1,2]$. It is diagnosed on the basis of a combination of patient-reported symptomswhich commonly include nausea, vomiting, early satiety, postprandial fullness, bloating, and upper abdominal pain-and an objective evaluation of gastric emptying [1-5]. Gastroparesis may be idiopathic or associated with diabetes. It has been estimated to affect up to $40 \%$ of patients with type 1 diabetes and $30 \%$ of patients with type 2 diabetes [6]. Diabetic gastroparesis (DGP) is hypothesized to be caused by impaired neural control of gastric motility, and vagal neuropathy is also a prominent feature $[1,7]$. Patients with DGP may experience slowed gastric emptying and altered absorption of oral hypoglycemic drugs [7].

Gastroparesis is associated with clinical complications including malnutrition, esophagitis, and Mallory-Weiss tears [7], and may result in adverse impacts on patients' lives, such as decreased social interaction, diminished work functioning, and anxiety or depression $[1,8]$. Further, as with other chronic GI disorders, the impact of gastroparesis may be underappreciated; the prevalence may be underreported because of, in part, underdiagnosis; and the long-term consequences of gastroparesis are an opportunity for further study [9].

In 2015, the Food and Drug Administration (FDA) released draft guidance for industry with recommendations regarding the clinical evaluation of medications to treat gastroparesis [7]. On the basis of a review of the literature, the guidance specifies five clinically significant signs and symptoms of gastroparesis: nausea, vomiting, early satiety, postprandial fullness, and upper abdominal pain. The guidance also concludes that currently there is no comprehensive patient-reported outcome measure that is appropriate to support labeling claims pertaining to the treatment of gastroparesis.

A recently completed phase 2 clinical trial to assess the efficacy and safety of a new guanylate cyclase $\mathrm{C}$ receptor agonist treatment for DGP provided an opportunity for in-depth exploration of patients' symptoms and perceptions of treatment (NCT02289846) [10]. Patients eligible for participation in the clinical trial had diagnosed DGP (type 1 or 2) without any history or evidence of mechanical obstructions, reported DGP symptoms for at least 3 months before the trial, and had evidence of delayed gastric emptying via scintigraphy or breath test. Exclusion criteria ensured that patients with other GI diseases, taking medications, or having undergone surgical procedures that may have confounded the assessment of gastroparesis symptoms were ineligible for the trial. An aim of the study was to gather patient input by conducting qualitative pretreatment and end-of-treatment interviews with trial participants regarding the DGP symptoms they experienced before and during treatment to inform either the modification of an existing DGP symptom severity measure or the development of a novel instrument. Specifically, the objectives of the patient interviews were to explore patients' experiences with DGP and identify DGP symptoms most important to treat; to inform the measurement strategy for future clinical trials in DGP; and to complement quantitative data collected during the trial via patient-reported outcome instruments, including a patient-completed diary (the primary measure of change in symptom severity). Conducting interviews in the context of a clinical trial provided the opportunity to efficiently gather input from a large number of patients and to characterize, in detail, the DGP patient experience.

\section{METHODS}

\section{Study Design and Population}

This multisite, qualitative study of individuals (18 years or older) participating in the placebocontrolled, double-blinded phase 2 clinical trial was conducted through individual telephone interviews. Study site staff at each clinic invited all clinical trial participants to participate in the interviews at enrollment. At screening, clinic staff explained the purpose and procedures associated with the patient interviews at the pretreatment visit (PTV) and end-of-treatment visit (EOTV) and obtained explicit consent from 
patients who wished to participate in the interviews. All procedures followed were in accordance with the ethical standards of the responsible committee on human experimentation (institutional and national) and with the Helsinki Declaration of 1964, as revised in 2013. Informed consent was obtained from all patients who participated in the study.

Two female authors with extensive qualitative research experience (CME and SEF) conducted all interviews by telephone. During the PTV and EOTV, study site staff took interview participants to a private location within the clinic, called the designated interviewer to initiate each interview, and then left the room. To supplement the interviewers' field notes and facilitate analysis, the interviews were audio recorded and transcribed. On completion of the interview, each participant was provided with an honorarium.

The target sample for the patient interviews included all trial participants who consented to be interviewed at the PTV and EOTV.

\section{Interview Methods}

Each interview was conducted with use of a semistructured interview guide and lasted approximately $60 \mathrm{~min}$. The interviewers had access to only a unique clinical trial identification number for each interview participant; no personally identifiable data or detailed demographics were collected.

The PTV interviews began with introductions, an overview of the interview objectives, and a description of the interview process. The interviewers (CME and SEF) then asked general, open-ended questions designed to identify the DGP symptoms relevant to each participant. More targeted questions followed to ensure the reporting of a comprehensive set of DGP symptoms and to elicit patients' perceptions and descriptions of symptom severity, the impact of DGP on patients' lives, the bothersomeness (0-10 scale, 10 being the most bothersome) and relative importance of each symptom, and what patients would need to experience to feel that a medication was working for them. If specific symptoms associated with gastroparesis in the literature and/or in existing patient-reported outcome measures were not mentioned spontaneously, interview participants were asked if they had experienced them. Interview participants were also asked to describe how their lives were impacted by their DGP symptoms.

The EOTV interviews focused on patients' experiences during the clinical trial, particularly any symptoms that had changed (regardless of treatment assignment); participants were also asked about the timing and importance of any changes they experienced. If none were mentioned spontaneously, interview participants were asked if they had experienced a change in any of the symptoms they reported during the PTV interview. In addition, the Gastroparesis Cardinal Symptoms Index-Daily Dairy [11] was briefly reviewed during the interview; these results are not reported herein.

\section{Data Analysis}

Analysis of the interview data was guided by a content analytic plan developed before conduct of the interview, was completed primarily by the interviewers (CME and SEF), and was facilitated by the use of qualitative software (ATLAS.ti version 7.5; Thomas Muhr, Scientific Software Development, 2013). While the analysis plan included an initial coding structure focused on symptoms and impacts of DGP, new codes were added (as needed) throughout the analysis process to ensure comprehensive and systematic coding of concepts and reporting of themes and patterns in patients' descriptions of experiences before and during the clinical trial. Given the sampling plan (i.e., invitation of all clinical trial participants), the achievement of concept saturation was anticipated but not specifically monitored.

In addition to conceptual coding, each interview was rated by quality: "green" indicated that the interview participant was able to understand the interview questions and provide highly relevant data, "yellow" indicated that the interview participant was able to understand most interview questions and provide relevant data, and "red" indicated that the interview 
participant did not understand most questions. In the analysis and reporting of frequencies, only unique mentions per code (concept) per patient were counted, and only the data resulting from interviews rated "green" or "yellow" were used.

\section{RESULTS}

\section{Sample Disposition}

A total of 78 individuals $(86.7 \%$ of the 90 enrolled trial participants [10]), recruited across 14 clinical sites, opted to participate in the interview study. The reasons for nonparticipation (among the 12 trial participants who declined to be interviewed) were not collected. The 78 PTV interviews were conducted between November 2014 and October 2015; 51 of these patients also completed an EOTV interview between January 2015 and November 2015. The reasons for attrition generally paralleled those in the clinical trial, including failure to meet randomization criteria and loss to follow-up. Among the PTV interviews, 73 interview transcripts were coded "green" or "yellow" and thus were included in the PTV data analysis. The five interview transcripts coded "red" came from patients for whom English appeared to be a second language. All 51 EOTV interview transcripts were included in the EOTV data analysis.

\section{Pretreatment Diabetic Gastroparesis Symptoms}

Table 1 presents the potentially unique DGP symptoms reported by the 73 PTV interview participants, either spontaneously or in response to probing; Table 2 presents selected verbatim statements from patients describing frequently reported symptoms. Bloating, stomach fullness, upper abdominal pain, vomiting, constipation, and heartburn or reflux emerged as major themes: each was reported spontaneously by more than 50\% of the PTV interview participants. Participants generally described experiencing symptoms of DGP daily or almost daily, and described an increasing intensity of symptoms throughout the day. Participants reported several ways in which DGP affected their lives (Table 3).

When asked to report their most bothersome DGP symptom, participants identified a total of 15 DGP symptoms (Table 4). Bloating was most frequently reported as participants' most bothersome symptom, followed by upper abdominal pain and nausea. The symptoms reported spontaneously by at least $50 \%$ of the 73 PTV interview participants and identified as most bothersome included bloating, upper abdominal pain, constipation, stomach fullness, heartburn or reflux, and vomiting; nausea was also included among the most bothersome symptoms by a substantial proportion (49.3\%) of PTV interview participants. Bloating was also most commonly identified as a symptom that would need to improve for participants to conclude that a DGP medication was working (Table 4).

\section{Stomach Fullness}

Across the 73 PTV interviews, stomach fullness and related symptoms (i.e., early satiety, postprandial fullness, and loss of appetite) emerged as central DGP symptoms. Stomach fullness was reported by 68 participants. Participants commonly noted a general tendency to feel full throughout the day, not just after eating. They also reported eating less in attempts to minimize DGP symptoms, including early satiety and postprandial fullness. Although the definitions of early satiety and postprandial fullness are conceptually distinct, participants rarely described these as separate symptoms, and instead considered "stomach fullness" to encompass both early satiety and postprandial fullness.

\section{Nausea and Vomiting}

Nausea and vomiting were spontaneously reported by approximately half of the PTV interview participants (details in Table 1). In contrast, retching was rarely reported until participants were specifically asked about this symptom. Nausea, reported by 62 participants, was described as a frequent (i.e., daily or every other day), intermittent symptom. Unlike DGP symptoms such as stomach fullness and 
Table 1 Diabetic gastroparesis symptoms reported: spontaneous, probed, and total $(n=73)$

\begin{tabular}{llll}
\hline Symptom & Spontaneous reports & Probed reports & Total reports \\
\hline Bloating & $51(69.9 \%)$ & $17(23.3 \%)$ & $68(93.2 \%)$ \\
Stomach fullness & $43(58.9 \%)$ & $25(34.2 \%)$ & $68(93.2 \%)$ \\
Upper abdominal discomfort & $16(21.9 \%)$ & $50(68.5 \%)$ & $66(90.4 \%)$ \\
Nausea & $36(49.3 \%)$ & $26(35.6 \%)$ & $62(84.9 \%)$ \\
Early satiety & $30(41.1 \%)$ & $31(42.5 \%)$ & $61(83.6 \%)$ \\
Stomach or belly visibly larger & $35(47.9 \%)$ & $24(32.9 \%)$ & $59(80.8 \%)$ \\
Upper abdominal pain & $38(52.1 \%)$ & $19(26.0 \%)$ & $57(78.1 \%)$ \\
Postprandial fullness & $15(20.5 \%)$ & $41(56.2 \%)$ & $56(76.7 \%)$ \\
Loss of appetite & $22(30.1 \%)$ & $31(42.5 \%)$ & $53(72.6 \%)$ \\
Vomiting & $39(53.4 \%)$ & $5(6.8 \%)$ & $44(60.3 \%)$ \\
Retching & $7(9.6 \%)$ & $33(45.2 \%)$ & $40(54.8 \%)$ \\
Constipation & $39(53.4 \%)$ & $\mathrm{NA}$ & $39(53.4 \%)$ \\
Heartburn or reflux & $38(52.1 \%)$ & $\mathrm{NA}$ & $38(52.1 \%)$ \\
Gas & $35(47.9 \%)$ & $\mathrm{NA}$ & $35(47.9 \%)$ \\
Diarrhea & $28(38.4 \%)$ & $\mathrm{NA}$ & $28(38.4 \%)$ \\
Regurgitation & $22(30.1 \%)$ & $2(2.7 \%)$ & $24(32.9 \%)^{\mathrm{a}}$ \\
Cramping & $18(24.7 \%)$ & $\mathrm{NA}$ & $18(24.7 \%)$ \\
General abdominal pain/discomfort & $18(24.7 \%)$ & $\mathrm{NA}$ & $18(24.7 \%)$ \\
Lower abdominal pain & $12(16.4 \%)$ & $\mathrm{NA}$ & $12(16.4 \%)$ \\
Lower abdominal discomfort & $7(9.6 \%)$ & $\mathrm{NA}$ & $7(9.6 \%)$ \\
Stomach rumbling & $7(9.6 \%)$ & $\mathrm{NA}$ & $7(9.6 \%)$ \\
Accidents & $2(2.7 \%)$ & $2(2.7 \%)$ \\
\hline
\end{tabular}

If specific symptoms associated with gastroparesis in the literature and/or in existing patient-reported outcome measures were not mentioned spontaneously, interview participants were asked if they had experienced them: stomach fullness, being unable to finish a normal-sized meal, feeling excessively full after meals, loss of appetite, nausea, retching, vomiting, bloating, stomach visibly larger, upper abdominal pain, and upper abdominal discomfort. Italicized symptoms were systematically probed per the pretreatment visit interview guide.

$N A$ not applicable

a Although not systematically probed, this symptom was endorsed twice in response to probing.

bloating, which could last for extended periods (e.g., throughout the day), nausea was typically described as having a shorter duration (e.g., less than $1 \mathrm{~h}$ ).

Vomiting, reported by 44 participants, was consistently described as a severe and impactful symptom that, for most, occurred less frequently than their other symptoms. Participants also reported a range of vomiting severity (often defined by the number of times it occurred and the amount of food expelled). Participants who reported vomiting commonly indicated that they were likelier to vomit on days when their other symptoms were more 
Table 2 Selected verbatim descriptions of diabetic gastroparesis symptoms reported or endorsed by $60 \%$ or more of pretreatment interview participants $(n=73)$

\begin{tabular}{|c|c|}
\hline Symptom & Verbatim statements \\
\hline \multirow[t]{3}{*}{ Bloating/distention } & $\begin{array}{l}\text { I have bloat all the time. All the time. Yep, it's like my stomach swell up like, kind of look like } \\
\text { you're pregnant, you know? }\end{array}$ \\
\hline & $\begin{array}{l}\text { It's just like it's swollen.... It feels, it's very uncomfortable. Some days it's not as bad as others, and } \\
\text { then when it's really bloated, distended, and hard, it's really uncomfortable, because your stomach } \\
\text { is tight }\end{array}$ \\
\hline & $\begin{array}{l}\text { Well, that's more of the bloating...so full that you can hardly move, and that's when I feel like my } \\
\text { stomach is really pushed out there and really at its limits }\end{array}$ \\
\hline \multirow[t]{3}{*}{ Stomach fullness } & $\begin{array}{l}\text { I can't eat well. I get full real easy.... And I feel fullness all day long. Like, I ate this morning, but I } \\
\text { still feel the food in my stomach }\end{array}$ \\
\hline & $\begin{array}{l}\text { I also have a feeling like a fullness even sometimes up into like almost my heart area.... I mean } \\
\text { sometimes I just feel like I've got to take that off, take my bra off and try to breathe... at the end } \\
\text { of the day, I feel like I'm about to bust open }\end{array}$ \\
\hline & $\begin{array}{l}\text { [How would you describe a worsening of stomach fullness?] A 10. Sometimes it's a full feeling, and } \\
\text { sometimes it's just not being interested in what I'm eating. Like the thought of eating makes me } \\
\text { sick... [Is that related to the fullness?] Yes }\end{array}$ \\
\hline \multirow[t]{3}{*}{ Early satiety } & $\begin{array}{l}\text { Typically, on my plate I may eat a tablespoon of peas and mashed potatoes, and I may even have } \\
\text { not even a fourth [of a] piece of meat, and I'm done }\end{array}$ \\
\hline & $\begin{array}{l}\text { Well, frankly I don't know how to describe it other than when you eat a big meal, you feel really } \\
\text { full. That happens to me when I eat a very small meal }\end{array}$ \\
\hline & After I have a few bites, it feels like...I had too much to eat when I know I've just had a few bites \\
\hline \multirow[t]{2}{*}{ Postprandial fullness } & $\begin{array}{l}\text { If I sit down to eat like, you know, you'll have a salad, you'll have a vegetable and you'll have a meat } \\
\text { and a drink. If I combined all of that together and I eat all of that, then say like } 20 \text { minutes or } \\
\text { something like that, I am miserable afterwards }\end{array}$ \\
\hline & $\begin{array}{l}\text { It's a very uncomfortable feeling, you feel really stuffed. And then you start burping a lot like your } \\
\text { body is trying to make room }\end{array}$ \\
\hline \multirow[t]{3}{*}{ Nausea } & Like, I feel sick that I want to throw up and really can't function because I'm that sick \\
\hline & $\begin{array}{l}\text { I try to eat something small, and just like one bite, sometimes I take one bite and have to head } \\
\text { straight to the bathroom to get rid of it to throw it up. I could take a bite of something and just } \\
\text { have the nausea happen all of a sudden }\end{array}$ \\
\hline & $\begin{array}{l}\text { When I'm feeling nauseous, I know I'm going to throw up. And it's not like reflux or anything like } \\
\text { that. I can tell the difference.... Higher up in my stomach, you know, I just know something is } \\
\text { there that I'm going to throw up }\end{array}$ \\
\hline
\end{tabular}


Table 2 continued

\begin{tabular}{|c|c|}
\hline Symptom & Verbatim statements \\
\hline \multirow[t]{3}{*}{ Vomiting } & $\begin{array}{l}\text { I couldn't hold any food down. It would go down and it would just stay there and stay there and } \\
\text { stay there until finally I would just go and throw it up }\end{array}$ \\
\hline & $\begin{array}{l}\text { Well, I just had a queasy feeling in my upper stomach and it feels like, you know, the feeling is } \\
\text { rising upward and I open my mouth and things come out }\end{array}$ \\
\hline & When you're throwing up food that's just sitting in your stomach or food that hasn't digested \\
\hline \multirow[t]{3}{*}{$\begin{array}{l}\text { Abdominal pain/ } \\
\text { discomfort }\end{array}$} & $\begin{array}{l}\text { Almost every day, you know, after I eat my stomach gets hard. It hurts. It felt like somebody was } \\
\text { just sticking you with something. I mean sticking a knife in your gut, and then at just other times } \\
\text { it felt like an open wound, that burning feeling that it gets }\end{array}$ \\
\hline & $\begin{array}{l}\text { Top part of my stomach and in the middle part of my stomach by my navel. It feel like sharp pains, } \\
\text { like needle pains }\end{array}$ \\
\hline & $\begin{array}{l}\text { And in my upper abdominal area, like maybe around my diaphragm or whatever, I just kind of } \\
\text { have a dull pain there at all times.... It's like, and again I'll say, in my diaphragm area and that } \\
\text { high up, it feels heavy like there's a weight, you know }\end{array}$ \\
\hline \multirow[t]{2}{*}{ Loss of appetite } & $\begin{array}{l}\text { [Can you describe a worsening of your fullness?] Having no appetite at all. No appetite. Taking } \\
\text { one bite and being done. Eating chicken nuggets or something like that [inaudible] two or three } \\
\text { of them and I'm done. I'm just done }\end{array}$ \\
\hline & $\begin{array}{l}\text { I just feel really heavy and I hurt when I try to eat something or drink something else when I be } \\
\text { feeling full like that. I can't. Even if I feel, per se, hungry, I can't put more in. I have to just stop }\end{array}$ \\
\hline
\end{tabular}

Quotations were identified by participant number (not presented here).

severe and that they generally felt slightly better after vomiting.

\section{Bloating}

Bloating was spontaneously reported by more participants (51 of 73) than any other symptom. Descriptions of bloating were consistent across interview participants, including a heavy, tight, full, and/or uncomfortable feeling above the navel, often accompanied by the swelling or a visible expansion of the stomach area. Distention was typically described as a component of bloating rather than a separate symptom. In contrast, participants often described a close relationship between feeling overly full and bloating but considered these concepts distinct.

\section{Abdominal Pain/Discomfort}

All 73 PTV interview participants described some type of abdominal pain or discomfort (details in Table 1). Most participants used the terms "pain" and "discomfort" interchangeably, and reported the location (upper or lower abdomen) only when prompted; even when asked, some participants were unable to identify a specific location. Participants generally described abdominal pain (general, upper, and/or lower) as an intermittent symptom that typically worsened with eating and/or drinking. Upper abdominal pain was often associated with heartburn, whereas lower abdominal pain was more likely associated with altered bowel habits. Cramping was generally described as a specific type of abdominal pain, often associated with an impending bowel movement. Abdominal discomfort (regardless of location) was rarely described as a unique symptom; in fact, it was almost always mentioned in reference to other abdominal symptoms.

\section{Heartburn or Reflux}

Heartburn or reflux was reported by 38 of the 73 PTV interview participants. Participants 
Table 3 Impacts of diabetic gastroparesis $(n=73)$

\begin{tabular}{lc}
\hline Impact & Participants \\
\hline Reluctant to leave the house & $27(37.0 \%)$ \\
Fatigue & $22(30.1 \%)$ \\
Food avoidance & $18(24.7 \%)$ \\
Sleep impacts & $12(16.4 \%)$ \\
Unable to eat as desired & $10(13.7 \%)$ \\
Difficult to maintain blood glucose level/ & $9(12.3 \%)$ \\
$\quad$ lack of glucose control & $8(11.0 \%)$ \\
Avoid going out to eat & $5(6.8 \%)$ \\
Negative work impacts & $4(5.5 \%)$ \\
\hline Dizziness &
\end{tabular}

Impacts reported by less than $5 \%$ of the sample included achiness $(n=3 ; 4.1 \%)$, headache $(n=3 ; 4.1 \%)$, and hair loss $(n=1 ; 1.4 \%)$.

described heartburn as a painful or uncomfortable sensation in the upper abdomen, chest, and/or throat that was commonly associated with acid reflux. Heartburn was commonly precipitated by eating (often regardless of the kind or quantity of food) and was frequently worse in the evening. Unlike participants' other DGP symptoms, which typically abated during the night, participants' heartburn-related symptoms often worsened when they were recumbent.

\section{Gas}

Various manifestations of gas (trapped gas, flatulence, and burping) were reported by nearly half (35) of the 73 PTV interview participants. Trapped gas was often associated with other symptoms such as bloating and abdominal pain, whereas flatulence was often associated with improvement of abdominal symptoms.

\section{Altered Bowel Habits}

Altered bowel habits were spontaneously reported by more than half (46) of the 73 interview participants. Constipation was reported more frequently than diarrhea (39 participants vs 28 participants); 21 participants reported both constipation and diarrhea.

\section{Impact of Diabetic Gastroparesis Symptoms}

PTV interview participants reported a variety of ways in which their lives were impacted by their DGP symptoms (Table 3 ). The most commonly reported impacts included reluctance to leave the house, fatigue, and food avoidance.

\section{Bothersomeness of Diabetic Gastroparesis and Symptom Improvement Indicative of Treatment Efficacy}

Interview participants were asked both to report their most bothersome DGP symptom and to rate the bothersomeness of that symptom. Participants included a total of 15 DGP symptoms among their most bothersome DGP symptoms (Table 4). Bloating was identified as participants' most bothersome symptom more frequently than any other symptom $(n=20$; mean bothersomeness rating 8.4), followed by upper abdominal pain $(n=12$; mean bothersomeness rating 8.6) and nausea ( $n=11$; mean bothersomeness rating 8.4). It is important to note, however, that all 73 participants did not experience the same constellation of DGP symptoms (e.g., more people reported bloating than vomiting).

Participants were also asked to report how they would know a DGP medication was working. Table 4 shows the frequency with which improvement in specific symptoms was reported as requisite evidence of a medication working. Improvement in bloating was the most frequently reported symptom improvement indicative of an effective medication. Other symptom improvements that were commonly reported (by ten or more participants) included nausea, constipation, stomach fullness, upper abdominal pain, and vomiting. When asked what kind of change in their symptoms they would need to see to keep taking the medication, most participants reported that a reduction in symptom intensity and frequency (although symptom elimination was ideal) that would allow them to eat normally would be considered an improvement. 
Table 4 Symptoms reported spontaneously, selected as most bothersome, and indicative of a diabetic gastroparesis (DGP) medication working $(n=73)$

\begin{tabular}{|c|c|c|c|}
\hline Symptom & Spontaneous reports ${ }^{\mathrm{a}}$ & $\begin{array}{l}\text { Selected as most } \\
\text { bothersome } e^{b, c}\end{array}$ & $\begin{array}{l}\text { Indicative of DGP } \\
\text { medication working } \mathrm{d}, \mathrm{e}\end{array}$ \\
\hline Bloating & $51(69.9 \%)$ & $20(27.4 \%)$ & $36(49.3 \%)$ \\
\hline Stomach fullness & $43(58.9 \%)$ & $5(6.8 \%)$ & $14(19.2 \%)$ \\
\hline Constipation & $39(53.4 \%)$ & $8(11.0 \%)$ & $15(20.5 \%)$ \\
\hline Vomiting & $39(53.4 \%)$ & $5(6.8 \%)$ & $10(13.7 \%)$ \\
\hline Upper abdominal pain & $38(52.1 \%)$ & $12(16.4 \%)$ & $13(17.8 \%)$ \\
\hline Heartburn or reflux & $38(52.1 \%)$ & $5(6.8 \%)$ & $7(9.6 \%)$ \\
\hline Nausea & $36(49.3 \%)$ & $11(15.1 \%)$ & $16(21.9 \%)$ \\
\hline Gas & $35(47.9 \%)$ & $4(5.5 \%)$ & $6(8.2 \%)$ \\
\hline Diarrhea & $28(38.4 \%)$ & $2(2.7 \%)$ & $6(8.2 \%)$ \\
\hline Regurgitation & $22(30.1 \%)$ & $1(1.4 \%)$ & $1(1.4 \%)$ \\
\hline Cramping & $18(24.7 \%)$ & $3(4.1 \%)$ & $4(5.5 \%)$ \\
\hline General abdominal pain/discomfort & $18(24.7 \%)$ & $1(1.4 \%)$ & - \\
\hline Upper abdominal discomfort & $16(21.9 \%)$ & $2(2.7 \%)$ & $4(5.5 \%)$ \\
\hline Postprandial fullness & $15(20.5 \%)$ & $1(1.4 \%)$ & - \\
\hline Retching & $7(9.6 \%)$ & $1(1.4 \%)$ & - \\
\hline
\end{tabular}

a Spontaneous reports provided only for symptoms selected among the most bothersome

b The sum of reports is more than 73 as some participants reported more than one most bothersome symptom

c Two participants were unable to respond, noting that all of their DGP symptoms were the most bothersome

d The sum of reports is more than 73 as some participants reported more than one symptom needing to improve

e Additional symptoms that participants reported would indicate that a DGP medication was working included stomach or belly visibly larger $(n=3 ; 4.1 \%)$, lower abdominal pain $(n=1 ; 1.4 \%)$, and regurgitation $(n=1 ; 1.4 \%)$

\section{Diabetic Gastroparesis Symptom Changes During the Trial}

Of the 51 EOTV interview participants (pooled across treatment arms), $44(86.3 \%)$ reported improvement in at least one DGP symptom. Most participants reported improvements in bloating, upper abdominal pain and discomfort, nausea, constipation, and the feeling of fullness. The additional key symptoms of early satiety and vomiting were also reported as improved by nearly half of the EOTV interview participants.

\section{Stomach Fullness}

Across the 51 EOTV interviews, stomach fullness (or feeling full) and related symptoms (i.e., early satiety, postprandial fullness, and loss of appetite) were frequently reported as improved. Specifically, more than half of the sample $(n=$ 28) reported an improvement in their stomach fullness, followed by a reduction in early satiety $(n=25)$, loss of appetite $(n=19)$, and postprandial fullness $(n=18)$. Improvements in fullness were consistently described as reductions in the severity or frequency with which participants felt unpleasantly full. Participants 
also noted that improvements in stomach fullness increased their desire to eat, as well as the ability to eat normal amounts without feeling excessively full (i.e., a boost in appetite and a reduction in early satiety and postprandial fullness), further supporting the strong relations among these symptoms.

\section{Nausea and Vomiting}

Most interview participants reported improvements in nausea $(n=31)$, including reductions in nausea severity and/or frequency. A few participants noted that their nausea went away completely. Just less than half of the interview participants reported improvements in their vomiting $(n=24)$. Participants commonly noted that improvement in vomiting was indicated by a reduction in vomiting frequency. However, some participants noted that their improvement was related to a reduction in vomiting severity, which was commonly described as a reduction in the amount of substance expelled or the force with which fluid/food left the body. Although less frequently reported, improvements in retching were reported by 21 participants; these improvements were commonly noted when participants were describing improvements in vomiting.

\section{Bloating}

Bloating was one of the symptoms most frequently reported as improving during the clinical trial $(n=34)$. Improvements in bloating were consistently described as a reduction of stomach swelling or size (stomach being visibly bigger, $n=22$ ).

\section{Abdominal Pain/Discomfort}

Upper abdominal pain was the other symptom most frequently reported as improving during the clinical trial $(n=34)$. Reductions in upper abdominal discomfort were also frequently reported $(n=30)$. Improvements in the remaining abdominal pain/discomfort symptoms (lower abdominal pain and discomfort, cramping, and general abdominal pain/discomfort) were reported with less frequency. Improvements in abdominal pain and discomfort were described as reductions in the intensity, frequency, and/or duration of these symptoms. Improvements in these symptoms were highly related (sometimes to the point of redundancy), with the alleviation of upper abdominal pain being the most salient and important to participants.

\section{Heartburn or Reflux}

A total of 18 participants described improvements in heartburn or reflux. Improvement in heartburn was commonly described as a reduction in the frequency with which participants experienced heartburn, particularly at night.

\section{Gas}

The presence of gas and its many manifestations (e.g., trapped gas and flatulence, as well as burping) was reported by 14 interview participants as improving.

\section{Bowel Habits}

Improvements in constipation were frequently reported and described by interview participants $(n=30)$ as an increase in the frequency of bowel movements, commonly accompanied by a reduction in straining and related abdominal symptoms (e.g., bloating, fullness, and discomfort). Two participants also reported an improvement in their diarrhea.

\section{Importance of Symptom Changes}

Forty-four of the 51 EOTV interview participants reported improvement in at least one symptom during the clinical trial. Of those 44 participants, 41 reported experiencing symptom improvement that was important or meaningful to them. Participants indicated that a reduction in symptoms often led to an improvement in their everyday lives (i.e., ability to go about their day feeling better and/or without as much fear of their symptoms). Among the 33 patients who reported improvements and were asked to identify their most important symptom improvement, improvements in bloating, upper abdominal pain, and constipation ( $n=12$ for each; some patients identified more than one most important abatement) were most frequently cited as the most important experienced during the trial. 


\section{DISCUSSION}

This study identified a comprehensive set of symptoms that are both bothersome and important to treat from the perspective of patients with DGP. The same symptoms likely would have been identified in qualitative research conducted outside the clinical trial setting. However, this study's larger sample size, the rigor with which participants were screened, and the details that were documented regarding participants' medical profile contribute to the robustness and generalizability of the results across the target patient population.

Nausea, vomiting (both frequency and severity), stomach fullness, bloating, upper abdominal pain, constipation, and heartburn were consistently identified and described as important symptoms. Specifically, these symptoms were most often reported spontaneously, identified as most bothersome, and cited as an indication that a DGP medication was working during the PTV interviews. Reductions in these symptoms during the trial were also described as important improvements by EOTV interview participants. Taken together, these results strongly suggest that, at a minimum, each of these symptoms should be measured in future DGP clinical trials.

The findings from this study also provide context for the interaction of DGP symptoms. For example, participants in this study rarely described abdominal discomfort as a unique symptom, but rather described it in reference to other abdominal symptoms (e.g., pain or bloating), suggesting that discomfort may overlap with or be caused by other symptoms. Although abdominal pain and bloating were among the DGP symptoms most frequently reported spontaneously, less than a quarter of the sample spontaneously reported abdominal discomfort. These findings suggest that abdominal discomfort may not be as salient to patients or as important to measure so as to obtain a comprehensive assessment of abdominal symptoms in future DGP clinical trials.

The results of this study are largely consistent with the FDA's draft gastroparesis guidance [7], which identifies nausea, vomiting, early satiety, postprandial fullness, and upper abdominal pain as the core gastroparesis symptoms. The primary point of departure is that, whereas most interview participants spontaneously reported stomach fullness as both bothersome and an important treatment target, they were far less likely to report early satiety and postprandial fullness as distinct symptoms. Instead, stomach fullness generally encompassed both early satiety and postprandial fullness among interview participants. The conceptual distinction between these symptoms lies in the time point at which the patient senses stomach fullness relative to when the patient stops stop eating, and eating behavior may vary for reasons unrelated to DGP symptom severity. Although the potential to assess early satiety and postprandial fullness separately should be explored further, it is possible that a single item addressing the feeling of stomach fullness may perform better than separate items.

Not only does some overlap across DGP symptoms exist, as suggested by the interview results, but the literature and the FDA acknowledge that the signs and symptoms of gastroparesis overlap considerably with those of other GI conditions (e.g., functional dyspepsia, irritable bowel syndrome, colonic motility) $[7,12]$. However, despite the exclusion of patients with GI conditions other than DGP from the clinical trial, bloating, constipation, and heartburn (none of which are considered core symptoms in the FDA's guidance) were common among interview participants. The interview results suggest that even if these three symptoms are not included in a primary efficacy end point in DGP treatment trials, they should likely be measured to demonstrate (at a minimum) a lack of worsening.

The results of the PTV and EOTV interviews also provide substantial insight into how to measure reduction in DGP symptoms. Modification of an existing measure or development of a new, DGP-specific symptom measure, informed by observations from this study, would appear necessary to support approval and labeling claims in future DGP trials. An additional objective of the current study was to complement and enrich quantitative trial data, 
providing insight into the impact of treatment on DGP symptoms. The patient diary and other patient-reported outcome measures administered during the trial offered a standard evaluation of treatment benefit, whereas these interviews offered an opportunity to more fully explore the impact of DGP symptoms and changes that occurred with treatment. For example, had unexpected changes occurred (reductions or worsening), the EOTV interview method may more likely have identified those changes given that the interviews were more open in nature than the diary assessments.

The results of this study should be considered within the context of its limitations. A potentially important limitation relates to the conduct of interviews via telephone rather than in person. In-person interviews typically encourage rapport and maximize participant engagement. In this context, because participants were enrolled in the study on an ongoing basis, in-person interviews would have been logistically challenging. However, the interviews were led by qualitative researchers who are experienced interviewers, and participants were generally forthcoming and engaged in the interviews despite the telephone-based method. As such, the study results likely would not have been substantially different had the interviews been conducted face-to-face. An additional limitation is that interview participants were self-selected clinical trial participants, and the results of the current study may not be generalizable to other patients with DGP. Finally, the interviews were conducted in English. The level of English-language proficiency required for participants to enter the trial may not have aligned with interview participants' ability to communicate richly about their trial experience.

\section{CONCLUSION}

Nausea, vomiting, stomach fullness (across its multiple manifestations), bloating, abdominal pain, constipation, and heartburn were reported as frequent and bothersome symptoms of DGP and provide targets for future DGP treatments. Modification of an existing DGP-specific symptom measure or development of a new measure should be considered for future research in DGP.

\section{ACKNOWLEDGEMENTS}

Sponsorship for this study, article processing charges, and the open access fee were funded by Ironwood Pharmaceuticals. All authors had full access to all of the data in this study and take complete responsibility for the integrity of the data and accuracy of the data analysis.

All named authors meet the International Committee of Medical Journal Editors (ICMJE) criteria for authorship for the manuscript, take responsibility for the integrity of the work as a whole, and have given final approval for the version to be published.

The authors gratefully acknowledge Melissa Villoldo, formerly of Ironwood Pharmaceuticals and currently of Revance Therapeutics, for her contributions to the design and operationalization of the study, and Diana Goss of RTI Health Solutions for the day-to-day management of the interview study. Kate Lothman of RTI Health Solutions provided medical writing services, which were funded by Ironwood Pharmaceuticals.

Disclosures. This study was performed under a research contract between Ironwood Pharmaceuticals and RTI Health Solutions. David S. Reasner is an employee of Ironwood Pharmaceuticals. Jennifer T. Hanlon is an employee of Ironwood Pharmaceuticals. Claire M. Ervin is an employee of RTI Health Solutions. Sheri E. Fehnel is an employee of RTI Health Solutions.

Compliance with Ethics Guidelines. All procedures followed were in accordance with the ethical standards of the responsible committee on human experimentation (institutional and national) and with the Helsinki Declaration of 1964, as revised in 2013. Informed consent was obtained from all patients who participated in the study. 
Data Availability. The datasets generated during and/or analyzed during the current study are not publicly available because no appropriate repository for the data exists.

Open Access. This article is distributed under the terms of the Creative Commons Attribution-NonCommercial 4.0 International License (http://creativecommons.org/licenses/by-nc/4. $0 /$ ), which permits any noncommercial use, distribution, and reproduction in any medium, provided you give appropriate credit to the original author(s) and the source, provide a link to the Creative Commons license, and indicate if changes were made.

\section{REFERENCES}

1. Parkman HP, Hasler WL, Fisher RS. American Gastroenterological Association medical position statement: diagnosis and treatment of gastroparesis. Gastroenterology. 2004;127:1589-91.

2. Camilleri M, Parkman HP, Shafi MA, Abell TL, Gerson L. Clinical guideline: management of gastroparesis. Am J Gastroenterol. 2013;108:18-37.

3. Camilleri M, Bharucha AE, Farrugia G. Epidemiology, mechanisms and management of diabetic gastroparesis. Clin Gastroenterol Hepatol. 2011;9:5-12.

4. Cherian D, Parkman HP. Nausea and vomiting in diabetic and idiopathic gastroparesis. Neurogastroenterol Motil. 2012;24(217-22):e103.

5. Hasler WL, Wilson LA, Parkman HP, Nguyen L, Abell TL, Koch KL, et al. Bloating in gastroparesis: severity, impact, and associated factors. Am J Gastroenterol. 2011;106:1492-502.

6. Parkman HP, Fass R, Foxx-Orenstein AE. Treatment of patients with diabetic gastroparesis. Gastroenterol Hepatol (N Y). 2010;6:1-16.

7. Food and Drug Administration. Gastroparesis: clinical evaluation of drugs for treatment. Draft guidance for industry. 2015. http://www.fda.gov/ ucm/groups/fdagov-public/@fdagov-drugs-gen/ documents/document/ucm 455645.pdf. Accessed 4 Oct 2016.

8. Soykan I, Sivri B, Sarosiek I, Kiernan B, McCallum RW. Demography, clinical characteristics, psychological and abuse profiles, treatment, and long-term follow-up of patients with gastroparesis. Dig Dis Sci. 1998;43:2398-404.

9. Food and Drug Administration. Patient-focused drug development: public meeting on functional GI disorders. 2015. http://www.fda.gov/ForIndustry/ UserFees/PrescriptionDrugUserFee/ucm 430885. htm. Accessed 1 Jan 2017.

10. ClinicalTrials.gov. Trial of IW-9179 in patients with diabetic gastroparesis (DGP). http://clinicaltrials. gov/ct2/show/NCT02289846?term=ironwood+ gastroparesis\&rank=1 (2016). Accessed 6 Dec 2016.

11. Revicki DA, Camilleri M, Kuo B, Norton NJ, Murray L, Palsgrove A, Parkman HP. Development and content validity of a gastroparesis cardinal symptom index daily diary. Aliment Pharmacol Ther. 2009;30:670-80.

12. Kolar GJ, Camilleri M, Burton D, Nadeau A, Zinsmeister AR. Prevalence of colonic motor or evacuation disorders in patients presenting with chronic nausea and vomiting evaluated by a single gastroenterologist in a tertiary referral practice. Neurogastroenterol Motil. 2014;26:131-8. 